\title{
Phobos' shape and topography models
}

\author{
K. Willner ${ }^{\mathrm{a}, *}$, X. Shi $^{\mathrm{b}}$, J. Oberst ${ }^{\mathrm{a}, \mathrm{b}}$ \\ a Technische Universität Berlin, Straße des 17. Juni 135, 10623 Berlin, Germany \\ b German Aerospace Center (DLR), Rutherfordstr. 2, 12489 Berlin-Adlershof, Germany
}

\section{A R T I C L E I N F O}

\section{Article history:}

Received 16 February 2013

Received in revised form

15 November 2013

Accepted 2 December 2013

Available online 18 December 2013

\section{Keywords:}

Phobos

Topography

Dynamic height

Shape model

\begin{abstract}
A B S T R A C T
The global shape and the dynamic environment are fundamental properties of a body. Other properties such as volume, bulk density, and models for the dynamic environment can subsequently be computed based on such models. Stereo-photogrammetric methods were applied to derive a global digital terrain model (DTM) with $100 \mathrm{~m} /$ pixel resolution using High Resolution Stereo Camera images of the Mars Express mission and Viking Orbiter images. In a subsequent least-squares fit, coefficients of the spherical harmonic function to degree and order 45 are computed. The dynamic models for Phobos were derived from a polyhedron representation of the DTM. The DTM, spherical harmonic function model, and dynamic models, have been refined and represent Phobos' dynamic and geometric topography with much more detail when compared to Shi et al. (2012) and Willner et al. (2010) models, respectively. The volume of Phobos has been re-determined to be in the order of $5741 \mathrm{~km}^{3}$ with an uncertainty of only $0.6 \%$ of the total volume. This reduces the bulk density to $1.86 \pm 0.013 \mathrm{~g} / \mathrm{cm}^{3}$ in comparison to previous results. Assuming a homogeneous mass distribution a forced libration amplitude for Phobos of $1.14^{\circ}$ is computed that is in better agreement with observations by Willner et al. (2010) than previous estimates. (c) 2013 Elsevier Ltd. All rights reserved.
\end{abstract}

\section{Introduction}

The shape of a body is a fundamental property that determines its volume, and sheds light on the knowledge of parameters such as bulk density, moments of inertia or the gravitational field. As a consequence, many models of Phobos' irregular shape were determined primarily based on image data gathered during current and past spacecraft missions to Mars (Duxbury et al., 2014; Witasse et al., 2014).

In the past, various kinds of shape models were used to represent Phobos and other planetary bodies (Table 1). A very simple shape approximation is the triaxial ellipsoid. This is only used for tasks which require little accuracy such as first observation planning or mission preparations. Other models, such as the spherical harmonic function model of higher degree and order, with e.g. added detail through local functions describing large craters (Duxbury, 1991), already represent Phobos' figure with much more detail. However, the resolution of these models is not high enough for applications such as cartographic mapping.

Based on image data of the two camera systems on board the Mars Express Mission (MEX), the High Resolution Stereo Camera (HRSC) and Super Resolution Channel (SRC), as well as the Viking orbiter cameras, two new shape models were derived to suit most needs. One is the global digital terrain model (DTM) with $100 \mathrm{~m} /$ pixel resolution

\footnotetext{
* Corresponding author.

E-mail address: konrad.willner@tu-berlin.de (K. Willner).
}

based on HRSC and Viking Orbiter image data. The DTM is used as a reference to compute ortho-rectified images. Such image maps are often applied in detailed analyses and are needed for accurately mapping surface features (Wählisch et al., 2010). On the other hand, a new spherical harmonic function model of degree and order 45 is provided that is used to compute volume estimates, bulk density, and rotation parameters (Willner et al., 2010). The latter is also used to derive a model for the forced libration amplitude assuming homogeneous mass distribution.

For small irregular bodies such as Phobos, processes of surface modification and material movement can be strongly influenced by the dynamical environment. Rotational and tidal forces can cause differences between dynamic and geometric topography due to their big influence. This is especially true for Phobos as it is orbiting relatively close to its central body (approximately $6000 \mathrm{~km}$ above the Martian surface), which causes large tidal forces in some areas of the body's surface. Furthermore, its synchronous rotation with a short period of $7.5 \mathrm{~h}$ causes high centrifugal forces. As an application of the shape model, a constant-density surface gravity model was developed for Phobos. Its dynamic topography is represented with dynamic height as well as dynamic slope.

\section{Methods for shape modeling}

Spacecraft observations of Phobos delivered essential information to model the elongated and rugged shape of this Martian 
Table 1

Types of shape models that were used to model Phobos' irregular body.

\begin{tabular}{|c|c|c|c|}
\hline Reference & Shape model type & Necessary observations & Base data \\
\hline Duxbury (1974) & \multicolumn{3}{|l|}{ Certainty in each axis $\pm 0.7 \mathrm{~km}$} \\
\hline Turner (1978) & $\begin{array}{l}\text { Plaster model } \\
\pm 1 \mathrm{~mm} \text { on the model relate to } \pm 60 \mathrm{r}\end{array}$ & $\begin{array}{l}\text { Light condition and camera position } \\
\text { simulation } \\
\text { ace }\end{array}$ & $\begin{array}{l}25 \text { Mariner } 9 \text { TV camera } \\
\text { images }\end{array}$ \\
\hline Thomas (1989) & \multicolumn{3}{|c|}{ $\pm 0.1 \mathrm{~km}$ to $\pm 0.4 \mathrm{~km}$ for the axes of the triaxial ellipsoid } \\
\hline Simonelli et al. (1993) & \multicolumn{3}{|c|}{$\begin{array}{l}2 \text { degree grid spacing; } \\
\text { Relative accuracy of radii between } \pm 50 \mathrm{~m} \text { (mean) and } \pm 200-400 \mathrm{~m} \text { (poor stereo coverage) }\end{array}$} \\
\hline Duxbury et al. (1991) & \multicolumn{2}{|c|}{ Developed to degree and order 8 with additional crater models } & Viking orbiter images \\
\hline Willner et al. (2010) & $\begin{array}{l}\text { Global digital terrain model } \\
100 \mathrm{~m} / \text { pixel resolution; relative accura }\end{array}$ & $\begin{array}{l}\text { Dense point cloud of conjugate points } \\
\text { tween } \pm 10 \mathrm{~m} \text { and } \pm 80 \mathrm{~m}\end{array}$ & HRSC images \\
\hline Willner et al. (2010) & $\begin{array}{l}\text { Spherical harmonic expansion model } \\
\text { Developed to degree and order } 17\end{array}$ & Dense distribution of ground control points & SRC images \\
\hline
\end{tabular}

moon. A variety of methods were applied leading to very good results at the time. The modeling improved as coverage and image resolution increased (see Table 1 for a historic record of Phobos Shape models). The very first global model was derived from Mariner 9 flyby data by plaster carving (Turner, 1978). This method was a predecessor of the shape from shading (SFS) approach that is applied on digital images today.

Another successful approach to derive information on Phobos' shape was to examine limb observations (see also Section 2.1). For Viking orbiter images, this proved to be more efficient than e.g. photogrammetric stereo reconstruction (PSR) since the number of limb observations was higher than the number of high resolution images suitable for PSR (see also Section 2.2).

\subsection{Limb modeling}

Limb observations provide a wealth of information as the locations of a large number of points along the limb can be determined. The accuracy of the limb measurements is limited by several factors. These are (a) the precision points of the limb can be determined which is in the range of $0.2-0.5$ pixels, (b) knowledge of the geometric distortion function of the acquiring imaging system of up to a few pixels, e.g. due to beam bending for vidicons (Dermott and Thomas, 1988), (c) the body-fixed coordinates of the limb points when the surface shape and spacecraft/body positions are not accurately known. Thomas (1989) derived ellipsoid models for Phobos, Deimos and other more regularly shaped bodies based on limb observations. The comparison with the solution of Duxbury and Callahan (1988), who derived the ellipsoid parameters through control network point information, showed very good agreement.

\subsection{Control point analysis}

Shape and topography models are usually based on information of object point coordinates of a large number of surface points. Control points have the special property that they can be re-identified in other data, hence, are visible surface features such as craters, boulders or parts there-of. With the advances in space exploration, such control point networks have been established for many bodies. The first control point network for Phobos was based on the image observations obtained during the Mariner 9 mission and consisted of 38 points (Duxbury, 1974) where the position of the points was known with an accuracy of approximately $\pm 1.3 \mathrm{~km}$. Consequently the control network was updated each time a data set of higher detail and accuracy was available (Duxbury and Callahan, 1989; Willner et al., 2010). See Oberst et al. (2014) for further details.

Due to the prerequisite that a control point needs to be a re-identifiable feature, the number of such points is usually sparse. Nonetheless, for shape modeling control points are of great value as they define a common reference to register different data sets against and determining orientation as well as libration angles.

\subsection{Image matching, conjugate points and DTM interpolation}

Image matching or image correlation is a well known technique to determine image coordinates of large numbers of surface points in two or more images. The objective is to find a large number of well constraint correlated points in the overlapping areas of at least two images to be able to numerically describe the imaged surface or object. In contrast to the control points, these conjugate points do not necessarily need to be re-identifiable such that automated processes can be used.

For planetary exploration data it is common practice to subsequently apply two different matching techniques. In a first step correlations are determined on a pixel accuracy level by applying normalized cross correlation (NCC) (see Heinrichs et al., 2007, for further details). To refine the results and reach a correlation within subpixel accuracy a least-squares routine is applied (Bethmann et al., 2010).

Both techniques compare gray values of equally sized pixel matrices to find the best correlation between the images. While the template pixel matrix is locked in its position within the template image the location of a very similar pixel matrix is searched for in a second image by moving the pixel matrix across the image within certain boundaries. It is desired to keep these boundaries small to avoid false correlations.

\subsection{Shape representations}

The derived 3D coordinates are commonly represented as DTMs or triangular irregular networks (TIN). 
DTMs are gridded representations and are often used for mapping purposes. A grid describes the surface or shape through a regular spaced matrix of height information. The matrix layout is defined by the underlying map projection and the elements are placed within this map-matrix according to the geocentric location of the respective surface area determined by the map projection formulas.

The extent of one height value, and thus the spatial resolution of the model, is given by the distance between the matrix elements. The height information can be given as height above a certain reference surface or as radius, e.g. of the geocentric coordinate. Depending on the completeness and spatial resolution of the input data, gridded 3D models, e.g. DTMs, can contain gaps. With increasing spatial resolution, surface details can be very well represented in a gridded shape model.

TINs have no fixed spatial resolution but use all 3D points that are connected to their nearest neighbors by triangles. The space between the 3D points can vary greatly, especially in areas where the surface coverage has low resolution. The triangles automatically interpolate across the space between the triangle vertices, no matter the separation distance.

A more general and continuous representation of the surface is the spherical harmonic function expansion. Radii can be obtained by fitting a spherical harmonic expansion to the control point radii. As with the TIN, the control points do not have to be evenly spaced. The harmonic expansion fills in areas of sparse data by smoothly interpolating the radii into these areas. The harmonic expansion accuracy increases with increased numbers of control points that fill in areas having less coverage. The more control points, the higher degree and order harmonic expansion can be determined. The distribution of control points is crucial in the accuracy of the expansion within areas of poor spatial resolution.

The surface is represented as a function of a number of coefficients. The expansion of a local radius can be evaluated at any body-fixed coordinate applying these coefficients and also provide the surface normal at the body-fixed coordinate.

These models are often used for computations of fundamental properties of the body that are constrained by its "shell" - such as the bulk density or moments of inertia tensor.

\section{HRSC data}

Since 2003 the MEX spacecraft has been in a highly elliptical polar orbit about Mars. With a distance to Mars of $11,000 \mathrm{~km}$ at its apoapsis, the spacecraft orbit reaches beyond the low orbit of Phobos at $9375 \mathrm{~km}$. This allows for close range observation of the Martian satellite approximately every 5 month. The MEX spacecraft hosts the High Resolution Stereo Camera which is a line scanning camera with a total of 9 lines mounted with an angular separation of up to $\pm 18.9^{\circ}$ with respect to the nadir pointing line. Only the two stereo, two photometry and the nadir channel images are used for the stereo reconstruction as they respond to the same spectra of light (rf. to Jaumann et al., 2007, for further details of the HRSC).

To determine a new, in comparison to Wählisch et al. (2010) yet more detailed, shape model for Phobos based on HRSC data, images were ortho-rectified onto a spherical harmonic function model (Willner et al., 2010) to minimize disparities between the images of the single channels of one flyby. A similar approach is followed when processing HRSC images of Mars to derive high resolution DTMs (Gwinner et al., 2009). To maximize the number of correlated points a grid search for the matching parameters-variation of search windows and quality factors for the correlation-was performed for each individual flyby image set considered (see Table 2).

The image coordinates of the correlated points are then used to determine the 3D coordinates of the surface points in object space by forward ray intersection.
Table 2

HRSC flybys considered for computation of the DTM. Confer Wählisch et al. (2014) for area covered by the different flybys.

\begin{tabular}{lcccc}
\hline \multirow{2}{*}{ Orbit no. } & Distance $(\mathrm{km})$ & \multicolumn{3}{l}{ Pixel scale $(\mathrm{m} /$ pixel $)$} \\
\cline { 3 - 5 } & & $\mathrm{ND}^{\mathrm{a}}$ & $\mathrm{S} 1 / \mathrm{S} 2^{\mathrm{b}}$ & $\mathrm{P} 1 / \mathrm{P}^{\mathrm{c}}$ \\
\hline \multirow{2}{*}{0756} & 148 & 6 & 13 & 12 \\
2780 & 612 & 24 & 26 & 50 \\
2854 & 886 & 39 & 43 & 80 \\
3310 & 566 & 24 & 25 & 50 \\
3843 & 650 & 26 & 27 & 54 \\
4307 & 579 & 23 & 24 & 47 \\
4340 & 679 & 28 & 29 & 57 \\
5851 & 92 & 4 & 4 & 8 \\
5870 & 352 & 14 & 15 & 29 \\
6906 & 527 & 21 & 22 & 42 \\
6916 & 518 & 20 & 22 & 43 \\
7478 & 478 & 19 & 20 & 39 \\
7488 & 588 & 24 & 25 & 48 \\
7915 & 111 & 4 & 5 & 9 \\
7926 & 287 & 11 & 13 & 24 \\
7937 & 475 & 19 & 20 & 39 \\
8974 & 100 & 4 & 4 & 8 \\
9574 & 360 & 14 & 15 & 29 \\
\hline
\end{tabular}

\footnotetext{
a Nadir channel.

b Stereo $1 /$ Stereo 2 channel.

c Photometries 1 and 2 Channel of the HRSC (cf. Jaumann et al., 2007).
}

Accurate knowledge of the camera position and orientation with respect to the body fixed frame of Phobos is required for this step. To make sure that different flyby data sets are referenced to a common coordinate frame a least-squares bundle block adjustment was carried out (Spiegel, 2007). Based on manually measured image coordinates of conjugate points between the images of different flybys and control points from the control point network (Willner et al., 2010) the exterior orientation parameters are adapted to minimize the residuals of the resulting object point coordinates.

\section{Results and implications}

\subsection{Gridded DTM}

The newly derived DTM shows Phobos' surface features in much more detail in comparison to e.g. the spherical harmonic shape model (Willner et al., 2010) or the previously computed DTM described in Wählisch et al. (2010). For the global data set a resolution of $100 \mathrm{~m}$ grid spacing appears to be suitable for displaying the DTM (see Fig. 1). However, locally a grid with a higher resolution could be chosen whilst introducing only little noise to the surface model. Large features like craters Stickney, Todd, Wendel, Gulliver are clearly visible while smaller craters with a depth of approximately $15 \mathrm{~m}$ and diameters as small as $480 \mathrm{~m}$ can very well be identified. In some regions groove features are recognizable. The point with the minimum radius derived from the stereophotogrammetric processing is located near the south pole $\left(86.45^{\circ} \mathrm{S}, 195.59^{\circ} \mathrm{W}\right)$ and has a radial distance to the center of figure of $7980 \mathrm{~m}$. The point with the maximum distance to the center is located at $2.83^{\circ} \mathrm{N}, 148.92^{\circ} \mathrm{W}$ with a radius of $13,910 \mathrm{~m}$.

The heights range from $-1235 \mathrm{~m}$ to $1500 \mathrm{~m}$ with respect to the triaxial ellipsoid with radii of $13.0 \mathrm{~km}, 11.4 \mathrm{~km}$, and $9.1 \mathrm{~km}$ (Willner et al., 2010), and range from $-2970 \mathrm{~m}$ to $3000 \mathrm{~m}$ with respect to a sphere of $11.1 \mathrm{~km}$ radius.

Fig. 2 shows the hypsogram for both reference bodies. The curve represents the cumulative sum of all heights (top and right axes). For better comparison heights were normalized for this plot. 

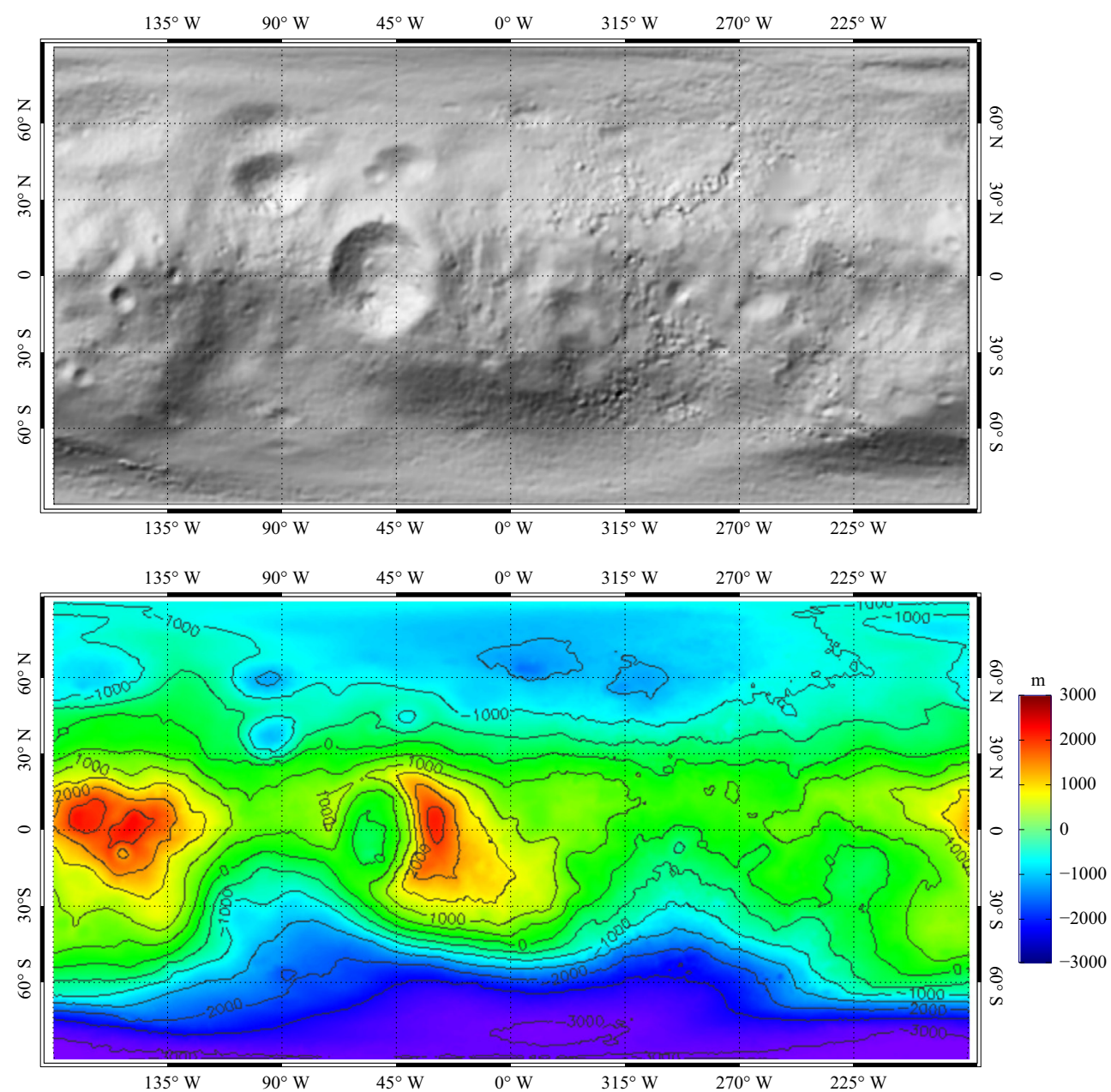

Fig. 1. Top: Equidistant shaded relief of the Phobos DTM. Bottom: Color coded map projection of the global DTM with heights above an 11.1 km sphere.

The curve for the reference sphere indicates that $5 \%$ of the height values are smaller than $-2670 \mathrm{~m}$ and another $5 \%$ of the height values are larger than $2700 \mathrm{~m}$. The remaining $90 \%$ of the heights appear to be evenly distributed as the almost constant inclination of the curve suggests. The curve for the triaxial ellipsoid indicates an even distribution of heights for most of the range. Only for the approximately $2 \%$ of the upper range heights a steep curve indicates a higher number of occurrences.

As shown in the percentage bar graphs, for heights above the ellipsoid there is an almost normal distribution while for heights above the sphere two peaks are prominent. These two peaks are right at the determined radii of two of the three axes of the triaxial ellipsoid. The largest axis, however, is not represented by a local maximum in the bar graph. This is due the low frequency of occurrences of large radii on Phobos and thus by the overall low percentage of radii near the $13 \mathrm{~km}$ mark.

\subsection{Ellipsoid- and spherical function shape}

Based on the current DTM the volume of Phobos was determined to be $5744 \mathrm{~km}^{3}$. When considering the spherical harmonic function model and applying discrete volume elements of $25 \mathrm{~m}$ edge length for the numerical integration a volume of $5741.5 \mathrm{~km}^{3}$ with an estimated error of $\pm 35 \mathrm{~km}^{3}$ (approximately $0.6 \%$ ) was computed. These values are slightly larger than but within the estimated errors of former volumes (Willner et al., 2010). A reason is that, even more information was available for this study, all data after orbit 5870 (Table 2) are new to the analysis and contribute significantly to details of the surface reconstruction in regions such as the south pole or the area between $90^{\circ}$ and $225^{\circ}$ West longitude, $30^{\circ}$ North and $50^{\circ}$ South latitude (cf. Fig. 1).

The spherical harmonic function model was further improved by introducing more than 5500 object point coordinates that were derived from the DTM analysis. The degree and order one coefficients initially indicated that the DTM coordinate frame is shifted with respect to the center of figure (COF) by $-384.71 \mathrm{~m}$ along the $x$-axis, $-306.04 \mathrm{~m}$ along the $y$-axis, and $206.71 \mathrm{~m}$ along $\mathrm{Z}$. The determination of this shift is an iterative process during which the control points are shifted according to the translation determined during the previous iteration step, before the harmonic expansion coefficients are computed anew.

Likewise, the rotations between the DTM coordinate frame and the principle axes frame were determined. As long as a significant rotational offset (larger than $0.05^{\circ}$ for any rotation axis) between the two frames was detected, the input point coordinates were rotated (see Eq. (1)) and the coefficients of the harmonic expansion were re-computed. Rotations of

$\phi=0.3378^{\circ}, \quad \omega=-0.4890^{\circ}, \quad \kappa=-177.8992^{\circ}$,

do align the axes of the two frames. Here $\phi$ is the angle for the rotation about the $x$-axis, $\omega$ about the $y$-axis, and $\kappa$ about the $z$-axis. The order of the rotations to be applied when a vector $\vec{X}^{\prime}$ in the control network frame is to be transformed to the principle 


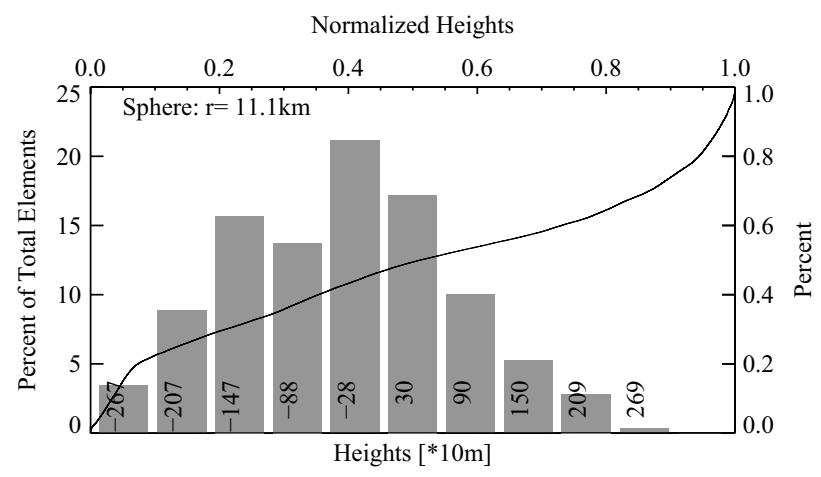

A

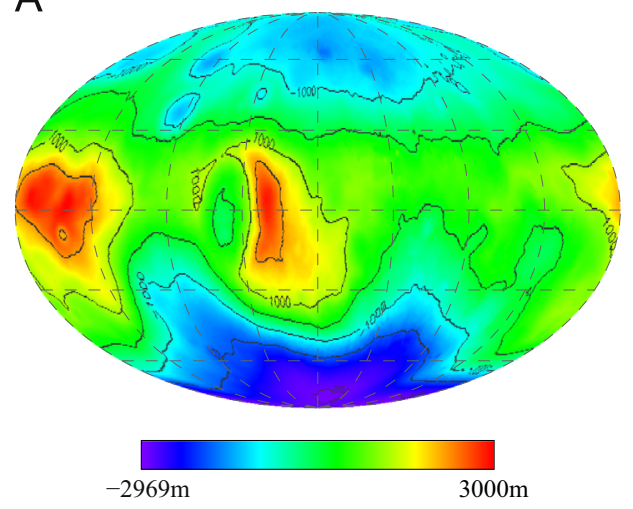

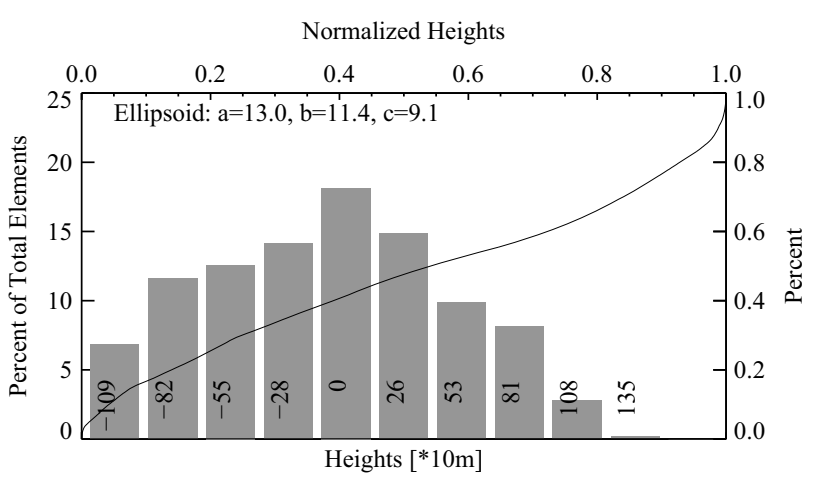

B

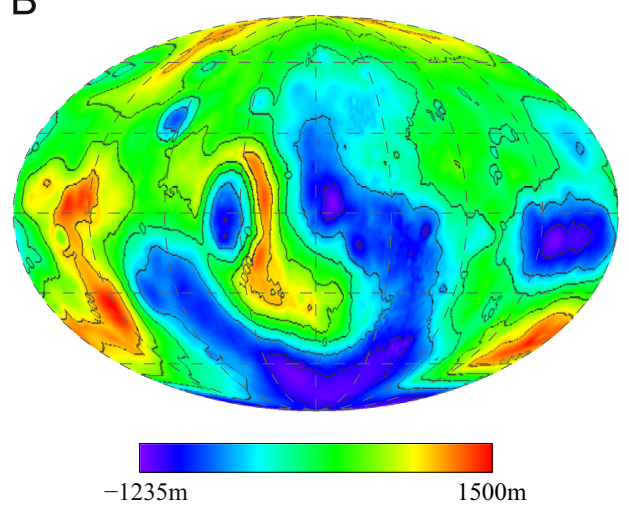

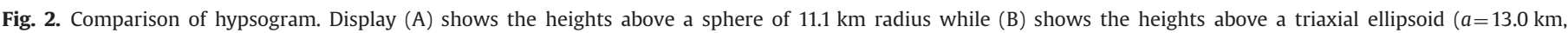

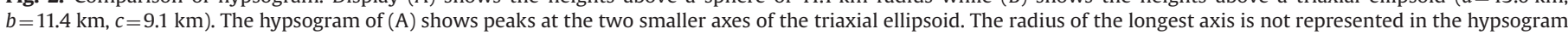
due to the low frequency of occurrences of radii of about $13 \mathrm{~km}$.

axes frame $\vec{X}$ is shown as

$\vec{X}=R_{z}(\kappa) R_{y}(\omega) R_{x}(\phi) \vec{X}^{\prime}$

The full transformation between the control point network/ DTM frame and the frame with the origin at the center of figure and axes aligned with the principle axes is given as follows:

$\vec{X}=R_{z y x}(\kappa, \omega, \phi) \vec{X}^{\prime}-\overrightarrow{\dot{x}}$,

where $\overrightarrow{\dot{x}}$ is the vector containing the shift between CoF and coordinate frame origin (see above).

The $2^{\circ}$ difference between the two frames is of no significance as the model frame is an arbitrary frame mainly defined by the orbit and Phobos' rotation model used for the computations. For this analysis Phobos position information was retrieved from the SPICE kernel MAR080 (Jacobson, 2008) and the rotational model is based on the values provided through the planetary constants kernel naif0010.tls.

All following results are based on the harmonic expansion model that is brought into agreement with the center of figure and the principle axes frame. As additional on-line material $\mathrm{CoF}$ centered and principle axes aligned coefficients of the spherical expansion are provided in two versions. TableA1.dat contains normalized coefficients. The normalization factor applied to the Associated Legendre Polynomials is

$\sqrt{\frac{(2 l+1)}{4 / \pi}} \sqrt{\frac{(l-m) !}{(l+m) !}} P_{l m}(\sin \Phi)$,

where $l$ and $m$ correspond to the degree and order of the spherical expansion, respectively, $P$ are the Associated Legendre Polynomials and $\Phi$ the observed latitude. For all coefficients in TableA2.dat the normalization factor has been applied and the coefficients are thus un-normalized.

The computed un-normalized coefficients to degree and order 45 indicate a mean radius for Phobos of $r_{0}=10.993 \mathrm{~km}$.

The ellipsoid parameters computed based on the spherical harmonic function model are slightly different in comparison to Willner et al. (2010) with the axial radii of $a=13.03 \mathrm{~km}$, $b=11.40 \mathrm{~km}$, and $c=9.14 \mathrm{~km}$.

Applying the volume derived from the spherical harmonic function model and the GM of $0.7127 \pm 0.0021 \times 10^{-3} \mathrm{~km}^{3} / \mathrm{s}^{2}$ observed through radio science (Andert et al., 2011) a bulk density of $1.860 \mathrm{~g} / \mathrm{cm}^{3}$ was determined.

After shifting and aligning the two reference frames and assuming a homogeneous mass distribution the principle moments of inertia (MoI) were computed to be

$A=0.3605, \quad B=0.4267, \quad C=0.4998$.

These values are unitless as they are normalized by $1 / M r_{0}$. The MoI differ only slightly from the ones published in Willner et al. (2010). However, the relation of the values influences the forced libration amplitude model. According to Eq. (3) (Chao and Rubincam, 1989)

$\theta_{(A)}=\frac{2 e}{1-\frac{1}{3 \hat{\gamma}}} \quad$ with $\hat{\gamma}=\frac{B-A}{C}$

where $\theta_{(A)}$ is the forced libration amplitude, $A \leq B \leq C$ are the principle moments of inertia (values as above), and $e=0.01511$ (Jacobson, 2010) is the orbital eccentricity of the body, the forced libration amplitude has a value of $\theta_{(A)}=-1.14^{\circ}$. While this value for the forced libration amplitude model is higher than the one determined in the previous analyses it is in better agreement with the observation of $\hat{\theta}_{(A)}=-1.24^{\circ}$ (Willner et al., 2010). 


\section{Surface gravity and dynamic topography}

\subsection{Methods}

The gravity field of a celestial body can be measured by analyzing spacecrafts' motion within the field using radio science data. To date, such gravity field models are available for only a limited number of solar system bodies. For Phobos, results from radio science data reduction merely show signals for low degree terms of its gravity field due to the short-arc data from flyby and an unfavorable observation geometry (Andert et al., 2011; Pätzold et al., 2014). Therefore, it is appropriate and practical to develop working models for the gravity field of Phobos using shape-based forward modeling method. This modeling approach is often practiced in the studies of small solar system bodies lacking recovered gravity information. In case of no affirmed knowledge of the internal structure, an assumption of constant density throughout the body is usually applied. In this study, the latest bulk density estimate of $1.860 \mathrm{~g} / \mathrm{cm}^{3}$ as mentioned above is adopted.

Different approaches can be used for forward modeling. With the shape model, it is shown that they all yield consistent results for Phobos (Shi et al., 2012). In order to better evaluate the dynamic topography, a detailed and continuous representation of the surface is required, for which we used the polyhedron method. By applying Delaunay triangulation, the latest DTM of Phobos is transformed into a polyhedron with 518,400 surface facets. Gravitational potential $U$ and gravitational acceleration a were then calculated on a surface grid with 0.5-degree interval using the equations from Werner and Scheeres (1996) (see Eq. (4)).

In Eqs. (4), $G$ is the gravitational constant, $\rho$ is the bulk density value, $\mathbf{r}_{F}$ is a vector from the field point to a certain surface polygon with $\hat{\mathbf{n}}_{F}$ as its outward-pointing unit surface normal, $\omega_{F}$ is the signed solid angle of this polygon viewed from the field point; $\mathbf{r}_{E}$ is a vector from the field point to one edge of this polygon with $\hat{\mathbf{n}}_{E}$ as its unit normal vector within the polygon plane, and $L_{E}$ is the integrated potential of this edge at the field point.

$U=\frac{1}{2} G \rho\left(\sum_{\text {faces edges }} \sum_{F} \cdot \hat{\mathbf{n}}_{F} \hat{\mathbf{n}}_{E} \cdot \mathbf{r}_{E} L_{E}-\sum_{\text {faces }} \mathbf{r}_{F} \cdot \hat{\mathbf{n}}_{F} \hat{\mathbf{n}}_{F} \cdot \mathbf{r}_{F} \omega_{F}\right)$

$\mathbf{a}=-G \rho\left(\sum_{\text {faces edges }} \sum_{F} \hat{\mathbf{n}}_{F} \hat{\mathbf{n}}_{E} \cdot \mathbf{r}_{E} L_{E}-\sum_{\text {faces }} \cdot \hat{\mathbf{n}}_{F} \hat{\mathbf{n}}_{F} \cdot \mathbf{r}_{F} \omega_{F}\right)$

Tidal and centrifugal forces are calculated using simplified orbital and rotational models. An averaged tidal effect is considered, using the mean orbital semi-major axis of $9375.0 \mathrm{~km}$ (Jacobson, 2010) as a fixed distance between Mars and Phobos. The centrifugal forces are calculated using a constant rotational rate together with the actual shape of Phobos.

By combining tidal and centrifugal forces together with the aforementioned self gravitational forces, the dynamical environment of Phobos can be assessed. Two parameters are used to describe the dynamic topography, dynamic height and dynamic slope. Dynamic height is the surface potential scaled by a reference gravity value with respect to a certain zero level of choice. It can be calculated according to the formula from Thomas (1993)

$H=\frac{V_{0}-V}{g_{0}}$

where $V$ is the total effective potential, including self-gravitational potential, centrifugal potential and tidal potential. The reference potential $V_{0}$ and reference gravity $g_{0}$ can be chosen arbitrarily. Here we used the mean surface potential value of $67.6 \mathrm{~m}^{2} / \mathrm{s}^{2}$ and the mean surface gravity value of $0.0084 \mathrm{~m} / \mathrm{s}^{2}$.

The dynamic slope is defined as the angle between the inwardpointing surface normal and the surface acceleration vector at a certain surface point. The surface normals are calculated using the polyhedron model of the shape.

\subsection{Results}

Fig. 4 shows a map of Phobos' dynamic height ranging from $-800 \mathrm{~m}$ to $800 \mathrm{~m}$. The signature of tidal effect can be clearly spotted with the depression in sub-Mars and anti-Mars areas. The highest value of dynamic height is found at $\left(273^{\circ} \mathrm{W}, 18^{\circ} \mathrm{N}\right)$ on the trailing side of Phobos. The point with the lowest value of dynamic height is found to be at $\left(188^{\circ} \mathrm{W}, 4.5^{\circ} \mathrm{S}\right)$. It is within one of the regions showing the strongest tidal and centrifugal forces.

Slopes on Phobos have as well a relatively large range of about $40^{\circ}$ (Fig. 3), which is typical for such irregular small bodies. Large craters can be easily identified in the slope map. In fact, crater walls show the largest values of slope, for example, the largest slope is found at $\left(60^{\circ} \mathrm{E}, 12^{\circ} \mathrm{S}\right)$ with a value of $40.6^{\circ}$ on the wall of the small crater Limtoc inside Stickney.

It is worth noticing that the slopes at many points on the west wall of crater Stickney are over $35^{\circ}$, possibly larger than the angle of repose for regolith-like materials. These steep slopes are also in accordance with the observed downslope movement in that area as discussed by Thomas et al. (2000).

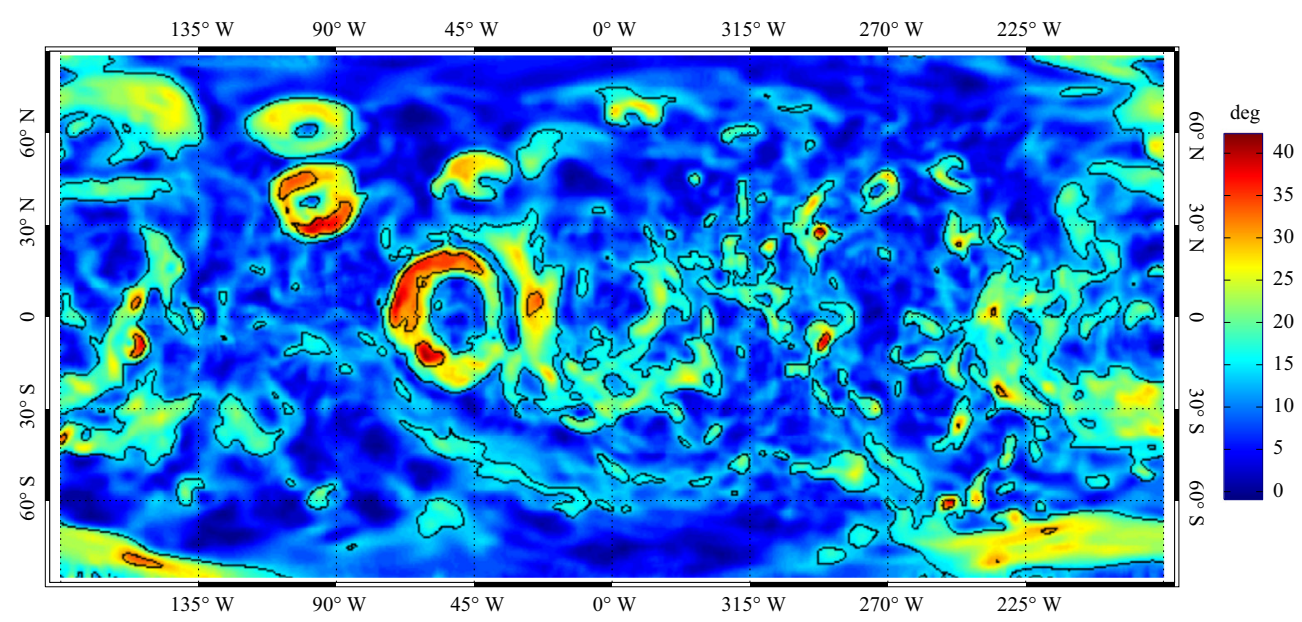

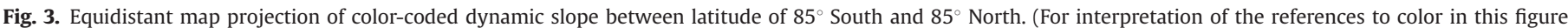
caption, the reader is referred to the web version of this paper.) 


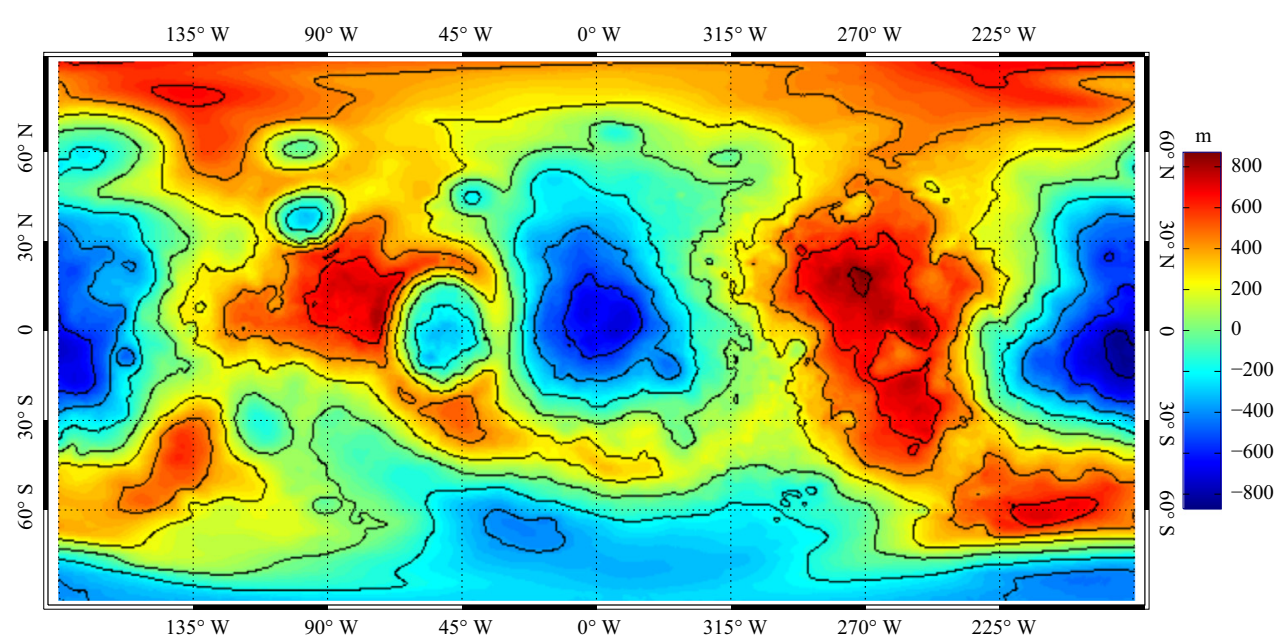

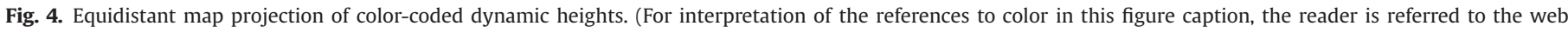
version of this paper.)
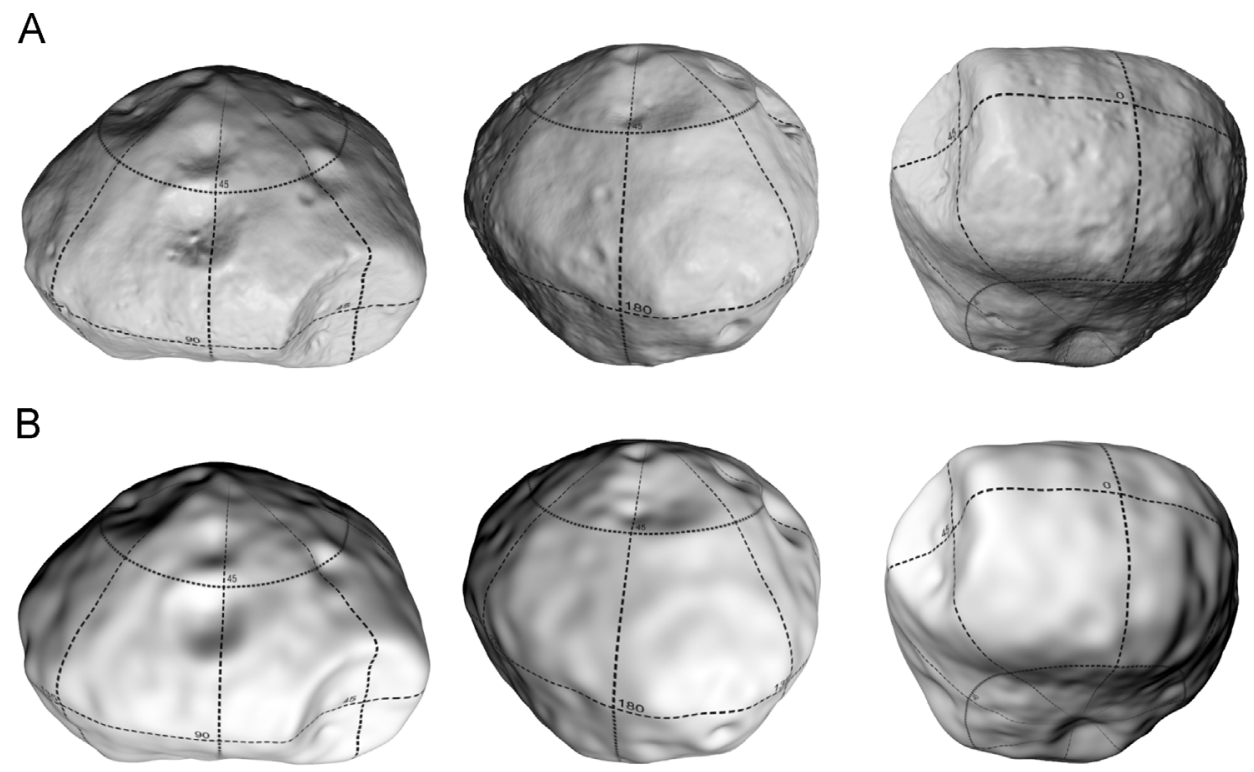

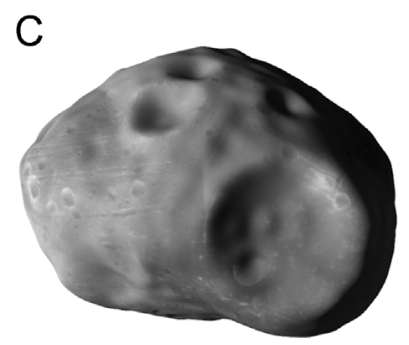

HRSC Mosaic

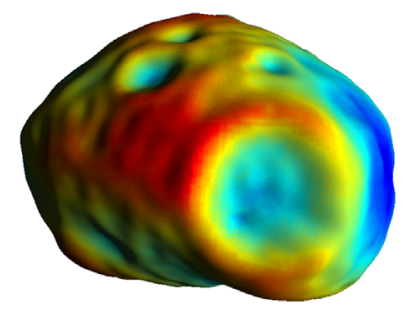

Dynamic Height

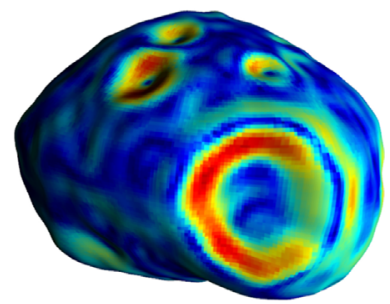

Dynamic Slopes

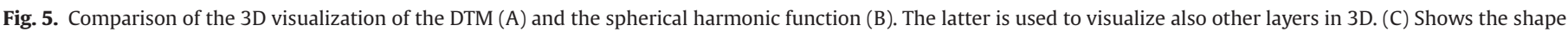

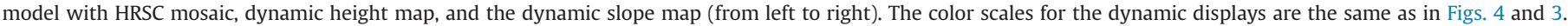

\section{Summary and conclusions}

With this study we provide new yet more detailed digital terrain and shape models for Phobos derived through photogrammetric stereo reconstruction from HRSC image data and by solving for the coefficients of the spherical harmonic function, respectively. Based on the new shape model (a) an updated volume estimate, (b) moments of inertia, (c) a model for the longitudinal forced libration amplitude, (d) a dynamic height model, and (e) a dynamic slope model were computed. A 3D model with the different layers, dynamic height and slope map, will be provided through the websites http://europlanet.dlr.de/Phobos and http:// www.planet.tu-berlin.de/?id=140044\&L=1 (see Figs. 5 and 6).

A global digital terrain model shows in comparison to the previous publication (Wählisch et al., 2010) significantly more detail though the same resolution is used. Single 'patches' of this 


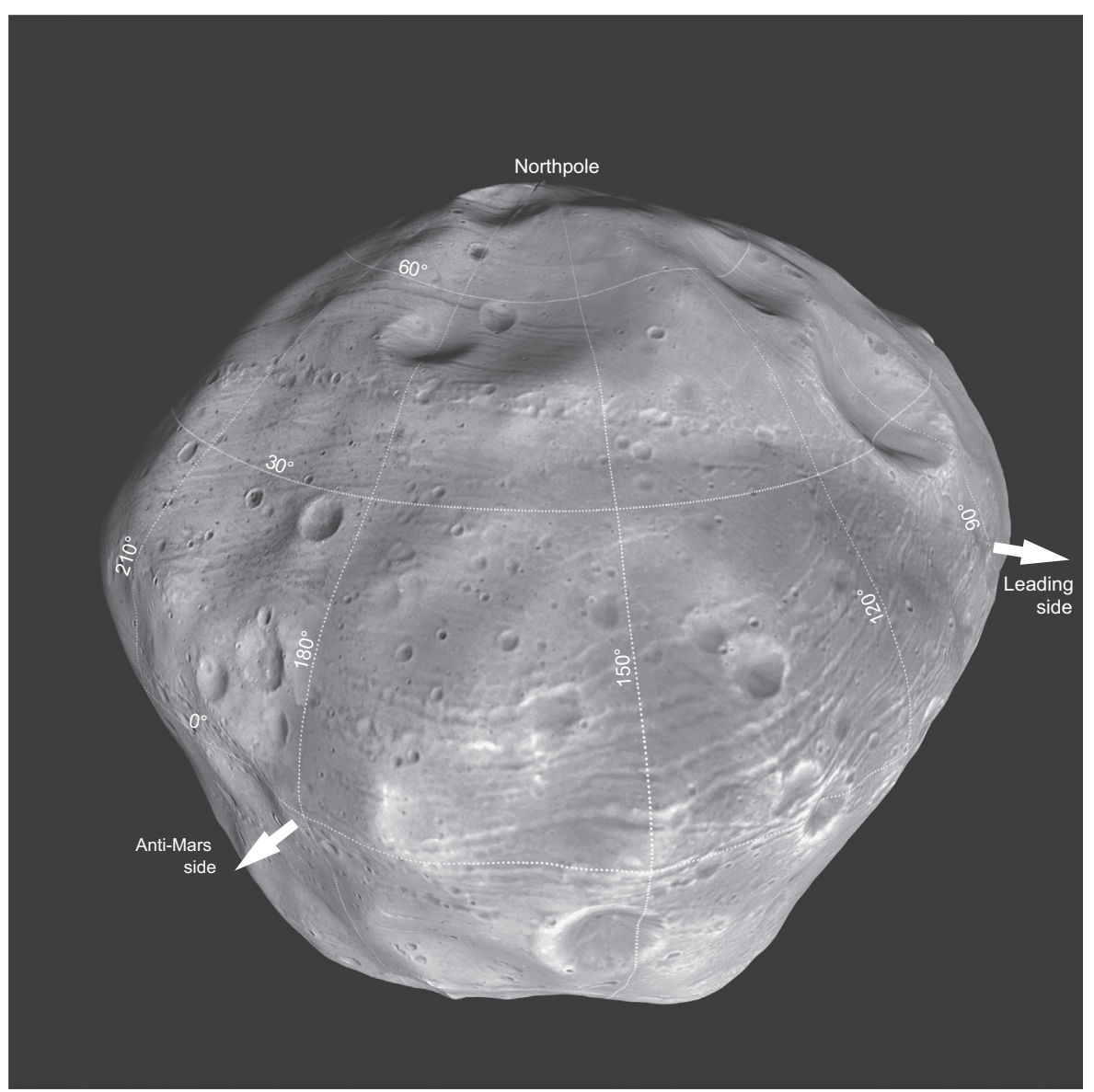

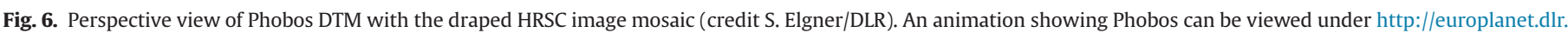
de/Phobos/video.

Table 3

Comparison of results from this study with results from Willner et al. (2010).

\begin{tabular}{lll}
\hline Property & Previous estimation & Current solution \\
\hline Radii & $13.00 \times 11.39 \times 9.07 \mathrm{~km}$ & $13.03 \times 11.40 \times 9.14 \mathrm{~km}$ \\
Volume & $5690 \pm 60 \mathrm{~km}^{3}$ & $5742 \pm 35 \mathrm{~km}^{3}$ \\
Moments of inertia (normalized) & \\
A, B, C & $0.3615,0.4265,0.5024$ & $0.3605,0.4267,0.4998$ \\
Forced libration amplitude & \\
Predicted & $1.1^{\circ}$ & $1.14^{\circ}$ \\
\hline
\end{tabular}

DTM can be produced with higher spatial resolution (e.g. Giese et al., 2005) depending on image resolution and quality of the image data that cover the region of interest.

Data points of the DTM were used to solve for the coefficients of the spherical harmonic functions to a degree and order 45 . Similar to the new DTM the shape model shows significantly more detail than the previous spherical harmonic function model (Willner et al., 2010). Even Limtoc, the largest crater within Stickney, can clearly be identified in this model (cf. Fig. 5).

Results from further analysis of this new shape model differ only slightly from the previous results (cf. Table 3 ). The prediction of the forced libration amplitude is in agreement with previous observations of Willner et al. (2010) but differs clearly from the studies of Oberst et al. (2014).

In Section 4.2 a translation and rotational discrepancy between the object point coordinate frame and the principle inertia frame is described. The authors see this offset and rotation of the two frames as a simple transformation between two independent frames. The realization of the coordinate frame for the DTM coordinates is founded on ephemeris as well as orientation models for Phobos (Jacobson, 2010) and the spacecraft. The latter is modified by the bundle block adjustment and thus somewhat arbitrary. In contrast the principle inertia frame is realized by the shape model and therefore well defined.

A new bulk density with the value of $1.860 \mathrm{~g} / \mathrm{cm}^{3}$ has been derived. Applying the law of error propagation with error values of $\sigma_{G M}=0.0021 \times 10^{-3} \mathrm{~km}^{3} / \mathrm{s}^{2}$ for the mass and $\sigma_{V}= \pm 35 \mathrm{~km}^{3}$ for the volume the one sigma error of the bulk density was determined to be $0.013 \mathrm{~g} / \mathrm{cm}^{3}$ or $0.7 \%$.

\section{Acknowledgments}

Cooperations supporting this work were initiated and furthered by the ISSI Bern Phobos and Deimos workshop series that took place between 2010 and 2012. The author has received funding from the European Union Seventh Framework Programme (FP7/2007-2013) under grant agreement no. 263466 - ESPACE for the research leading to these results. $\mathrm{X}$. Shi is supported by the DLR-DAAD Research Fellowship program.

We thank the HRSC Experiment Teams at DLR Berlin and Freie Universitaet Berlin as well as the Mars Express Project Teams at ESTEC and ESOC for their successful planning and acquisition of data as well as for making the processed data available to the HRSC team.

We acknowledge the effort of the HRSC Co-Investigator Team members and their associates who have contributed to this 
investigation in the preparatory phase and in scientific discussions within the Team.

\section{Appendix A. Supplementary material}

Supplementary data associated with this article can be found in the online version at http://dx.doi.org/10.1016/j.pss.2013.12.006.

\section{References}

Andert, T.P., Rosenblatt, P., Pätzold, M., Häusler, B., Tyler, G.L., 2011. The interna structure of Phobos and hints to its origin derived from Mars Express Radio Science observations. In: EPSC-DPS Joint Meeting 2011, p. 210.

Bethmann, F., Luhmann, T., Commission, V., Wg, V., 2010. Least-squares matching with advanced geometric transformation models. ISPRS Comm. V XXXVIII, Part 5, pp. 86-91.

Chao, B.F., Rubincam, D.P., 1989. The gravitational field of Phobos. Geophys. Res. Lett. 16 (August (8)), 859-862.

Dermott, S.F., Thomas, P.C., 1988. The shape and internal structure of Mimas. Icarus 73 (January), 25-65.

Duxbury, T., Wang, M., Herkenhoff, H., 1991. The figure of Phobos: an update from the PHOBOS mission. In: Lunar and Planetary Institute Conference Abstracts, Vol. 22. Lunar and Planetary Inst. Technical Report. pp. 327-+.

Duxbury, T.C., 1974. PHOBOS - control network analysis. Icarus 23 (October), 290-299.

Duxbury, T.C., 1991. An analytic model for the PHOBOS surface. Planet. Space Sci. 39 (February), 355-376.

Duxbury, T.C., Callahan, J.D., 1988. РНОВOS and Deimos astrometric observations from Viking. Astron. Astrophys. 201 (July), 169-176.

Duxbury, T.C., Callahan, J.D., 1989. PHOBOS and Deimos control networks. Icarus 77 (February), 275-286.

Duxbury, T.C., Zakharov, A., Hoffmann, H., Ball, A., 2014. History of spacecraft exploration 102, 9-17.

Giese, B., Oberst, J., Scholten, F., Hoffmann, H., Spiegel, M., Neukum, G., 2005. Ein hoch auflösendes Oberflächenmodell des Marsmondes Phobos. PFG Jahrg. 2005 (5), 439-444.

Gwinner, K., Scholten, F., Spiegel, M., Schmidt, R., Giese, B., Oberst, J., Heipke, C. Jaumann, R., Neukum, G., 2009. Derivation and validation of high-resolution digital terrain models from Mars Express HRSC-Data. Photogramm. Eng. Remote Sens. 75 (September (9)), 1127-1142.

Heinrichs, M., Rodehorst, V., Hellwich, O., 2007. Efficient semi-global matching for trinocular stereo. In: Stilla, U., Mayer, H., Rottensteiner, F., Heipke, C., Hinz, S. (Eds.), Proceedings of Photogrammetric Image Analysis (PIA 2007), Vol. 36, Part 3/W49A of International Archives of Photogrammetry, Remote Sensing and Spatial Information Science. Munich, pp. 185-190.

Jacobson, R.A., 2008. Ephemerides of the Martian Satellites-MAR080. IOM 343R08-006, JPL.
Jacobson, R.A., 2010. The orbits and masses of the Martian satellites and the libration of phobos. Astron. J. 139 (February), 668-679.

Jaumann, R., Neukum, G., Behnke, T., Duxbury, T.C., Eichentopf, K., Flohrer, J., Gasselt, S.v., Giese, B., Gwinner, K., Hauber, E., Hoffmann, H., Hoffmeister, A., Köhler, U., Matz, K.-D., McCord, T.B., Mertens, V., Oberst, J., Pischel, R., Reiss, D., Ress, E., Roatsch, T., Saiger, P., Scholten, F., Schwarz, G., Stephan, K., Wählisch, M., 2007. The high-resolution stereo camera (HRSC) experiment on Mars express: instrument aspects and experiment conduct from interplanetary cruise through the nominal mission. Planet. Space Sci. 55 (May), 928-952.

Oberst, J., Zubarev, A., Nadezhdina, I., Shishkina, L., Rambaux, N., 2014. The phobos geodetic control point network and rotation 102, 45-50.

Pätzold, M., Rosenblatt, P., Andert, T., Dehant, V., Jacobson, R., 2014. Phobos observed internal properties 102, 86-94.

Shi, X., Willner, K., Oberst, J., Ping, J., Ye, S., 2012. Working models for the gravitational field of Phobos. Sci. China G: Phys. Astron. 55 (February), 358-364.

Simonelli, D.P., Thomas, P.C. Carcich, B.T., Veverka, J., 1993. The generation and use of numerical shape models for irregular solar system objects. Icarus 103 (May), 49-61.

Spiegel, M., 2007. Kombinierte Ausgleichung der Mars Express HRSC Zeilenbilddaten und des Mars Global Surveyor MOLA DGM. Diss., Technische Universität München, Fakultät für Bauingenieur- und Vermessungswesen.

Thomas, P.C., 1989. The shapes of small satellites. Icarus 77 (February), 248-274.

Thomas, P.C., 1993. Gravity, tides, and topography on small satellites and asteroidsapplication to surface features of the Martian satellites. Icarus 105 (October), 326-344.

Thomas, P.C., Veverka, J., Sullivan, R., Simonelli, D.P., Malin, M.C., Caplinger, M., Hartmann, W.K., James, P.B., 2000. Phobos: regolith and ejecta blocks investigated with Mars orbiter camera images. J. Geophys. Res. 105 (June), 15091-15106.

Turner, R.J., 1978. A model of PHOBOS. Icarus 33 (January), 116-140.

Wählisch, M., Stooke, P.J., Karachevtseva, I.P., Kirk, R., Oberst, J., Willner, K., Nadejdina, I.A., Zubarev, A.E., Konopikhin, A.A., Shingareva, K.B., 2014. Phobos and Deimos cartography. Planet. Space Sci 102, 60-73, http://dx.doi.org/ 10.1016/j.pss.2013.05.012.

Wählisch, M., Willner, K., Oberst, J., Matz, K., Scholten, F., Roatsch, T., Hoffmann, H., Semm, S., Neukum, G., 2010. A new topographic image atlas of Phobos. Earth Planet. Sci. Lett. 294 (June), 547-553.

Werner, R.A., Scheeres, D.J., 1996. Exterior gravitation of a polyhedron derived and compared with harmonic and mascon gravitation representations of asteroid 4769 Castalia. Celest. Mech. Dyn. Astron. 65 (September), 313-344.

Willner, K., Oberst, J., Hussmann, H., Giese, B., Hoffmann, H., Matz, K., Roatsch, T. Duxbury, T., 2010. Phobos control point network, rotation, and shape. Earth Planet. Sci. Lett. 294 (June), 541-546.

Witasse, O., Duxbury, T., Chicarro, A., Altobelli, N., Andert, T., Aronica, A., Barabash, S., Bertaux, J.-L., Bibring, J.-P., Cardesin-Moinelo, A., Cichetti, A., Companys, V., Dehant, V., Denis, M., Formisano, V., Futaana, Y., Giuranna, M., Gondet, B., Heather, D., Hoffmann, H., Holmström, M., Manaud, N., Martin, P., Matz, K.-D., Montmessin, F., Morley, T., Mueller, M., Neukum, G., Oberst, J., Orosei, R., Pätzold, M., Picardi, G., Pischel, R., Plaut, J., Reberac, A., Voss, P.P., Roatsch, T., Rosenblatt, P., Remus, S., Schmedemann, N., Willner, K., Zegers, T., 2014. Mars express investigations of Phobos and Deimos. Planet. Space Sci. 102, 18-34. http://dx.doi.org/10.1016/j.pss.2013.08.002. 